\begin{tabular}{|c|l|}
\hline Title & Direct extraction of electron parameters from magnetoconductance analysis in mesoscopic ring array structures \\
\hline Author(s) & Sawada, A.; Faniel, S.; Mineshige, S.; Kaw abata, S.; Saito, K.; Kobay ashi, K.; Sekine, Y.; Sugiy ama, H.; Koga, T. \\
\hline Citation & $\begin{array}{l}\text { Physical Review B, 97(19), 195303 } \\
\text { https://doi.org/10.1103/PhySRevB.97.195303 }\end{array}$ \\
\hline Issue Date & 2018-05-08 \\
\hline Doc URL & http://hdl.handle.net/2115/70799 \\
\hline Rights & @2018A merican Physical Society \\
\hline Type & article \\
\hline File Information & PhysRevB.97.195303.pdf \\
\hline
\end{tabular}

Instructions for use 


\title{
Direct extraction of electron parameters from magnetoconductance analysis in mesoscopic ring array structures
}

\author{
A. Sawada, ${ }^{1}$ S. Faniel,${ }^{1,2}$ S. Mineshige, ${ }^{1}$ S. Kawabata,${ }^{3}$ K. Saito, ${ }^{4}$ K. Kobayashi,${ }^{5}$ Y. Sekine, ${ }^{6}$ H. Sugiyama,${ }^{7}$ and T. Koga ${ }^{1, *}$ \\ ${ }^{1}$ Graduate School of Information Science and Technology, Hokkaido University, Sapporo, Hokkaido 060-0814, Japan \\ ${ }^{2}$ ICTEAM/Winfab, Université catholique de Louvain, B-1348 Louvain-la-Neuve, Belgium \\ ${ }^{3}$ National Institute of Advanced Industrial Science and Technology, Tsukuba, Ibaraki 305-8568, Japan \\ ${ }^{4}$ Department of Physics, Keio University, Yokohama, Kanagawa 223-8522, Japan \\ ${ }^{5}$ Department of Physics, Graduate School of Science, Osaka University, 1-1 Machikaneyama, Toyonaka, Osaka 560-0043, Japan \\ ${ }^{6}$ NTT Basic Research Laboratories, NTT Corporation, 3-1 Morinosato-Wakamiya, Atsugi, Kanagawa 243-0198, Japan \\ ${ }^{7}$ NTT Device Technology Laboratories, NTT Corporation, 3-1 Morinosato-Wakamiya, Atsugi, Kanagawa 243-0198, Japan
}

(Received 30 March 2017; revised manuscript received 10 April 2018; published 8 May 2018)

\begin{abstract}
We report an approach for examining electron properties using information about the shape and size of a nanostructure as a measurement reference. This approach quantifies the spin precession angles per unit length directly by considering the time-reversal interferences on chaotic return trajectories within mesoscopic ring arrays (MRAs). Experimentally, we fabricated MRAs using nanolithography in InGaAs quantum wells which had a gate-controllable spin-orbit interaction (SOI). As a result, we observed an Onsager symmetry related to relativistic magnetic fields, which provided us with indispensable information for the semiclassical billiard ball simulation. Our simulations, developed based on the real-space formalism of the weak localization/antilocalization effect including the degree of freedom for electronic spin, reproduced the experimental magnetoconductivity (MC) curves with high fidelity. The values of five distinct electron parameters (Fermi wavelength, spin precession angles per unit length for two different SOIs, impurity scattering length, and phase coherence length) were thereby extracted from a single MC curve. The methodology developed here is applicable to wide ranges of nanomaterials and devices, providing a diagnostic tool for exotic properties of two-dimensional electron systems.
\end{abstract}

DOI: 10.1103/PhysRevB.97.195303

\section{INTRODUCTION}

Decades of research in quantum chaos [1-5] have revealed that scattering from geometric features in ballistic systems, such as open quantum dots, behaves in the same way as impurity scattering in bulk diffusive systems in terms of quantum interference phenomena. These phenomena include universal conductance fluctuations (UCFs) [1,2] and the weak localization/antilocalization (WL/WAL) effect [3,4,6-8]. On the basis of these studies, it has been proposed that the gatecontrollable Rashba spin-orbit interaction (SOI) $[9,10]$ can be studied quantitatively by considering the time-reversal quantum interference on ballistic square loop arrays fabricated in pristine semiconductor quantum wells (QWs) [11]. However, there has been only a qualitative success in this approach so far $[12,13]$.

The linearity between the Rashba SOI coefficient $\alpha$ and the averaged internal electric field $\left\langle E_{z}\right\rangle$, on the other hand, has been demonstrated experimentally using bulk two-dimensional electron systems (2DESs) [14], where $E_{z}$ is perpendicular to the heterointerfaces. We define the Rashba Hamiltonian to be $H_{\mathrm{R}}=\alpha\left\{k_{y} \sigma_{x}-k_{x} \sigma_{y}\right\}, \sigma_{x}$ and $\sigma_{y}$ being the Pauli spin matrices. The particular material system used in the series of studies was 10-nm-thick InAlAs/InGaAs/InAlAs QWs that are lattice matched to (001)InP. The Dresselhaus SOI [15] was revealed to

\footnotetext{
*koga@ist.hokudai.ac.jp
}

be negligible relative to the Rashba SOI in this system, unless $\alpha$ is carefully tuned to be zero by the gate voltage $\left(V_{\mathrm{g}}\right)$ [14].

In this study, we revisit our original idea that the electron properties including the Rashba SOI can be determined using the shape and size information of a nanostructure as a measurement reference [11]. We fabricated mesoscopic ring array (MRA) structures using the same pristine QW wafers as those used in the previous studies $[12,14]$, but paying a particular attention to the MRA shape so that the resultant electron orbitals become chaotic $[11,12,16]$. The trigger of this work was really an unexpected observation that the amplitude of the Altshuler-Aronov-Spivak- (AAS-) type oscillations in our MRA was diminished when the temperature was lowered from $\sim 1 \mathrm{~K}$ to the base temperature of the dilution refrigerator $(\sim 15 \mathrm{mK})$ in our preliminary experiment. The lack of quantitative models for the observed $\Delta \sigma(B)$ (oscillating component of the magnetoconductivity curve) prompted us to build one ourselves in this work.

MRAs provide an important test bed for exploring quantum phenomena relevant to charge and spin degrees of freedom [12,13,17-19]. The main advantage of MRAs over single Aharonov-Bohm- (AB-) type rings [20] lies in their tolerance of mesoscopic fluctuations, while maintaining other mesoscopic effects of electrons [17]. While the amplitudes of the regular $\mathrm{AB}$ oscillations (associated with the $h / e$ flux quantum) and the UCF are known to be suppressed according to $O(1 / \sqrt{N})(N=5408$ in our MRAs $)$ by ensemble averaging $[17,21]$, the amplitude of the AAS-type oscillations [22] is 
unaffected by ensemble averaging. This phenomenon shares the same physical origin as the WL/WAL effect [14,23-27]. We therefore expect to observe only the AAS-type oscillations (or the WL/WAL effect) in our measurement, where the regular $\mathrm{AB}$ and UCF effects are substantially diminished.

We investigate the magnetoconductivity (MC) of these samples at dilution refrigeration temperatures $(<1 \mathrm{~K})$ experimentally. It is found that the semiclassical billiard ball simulations can reproduce the measured MC oscillations of the MRA very accurately, including their fine structure and background components. Our simulations account for the time-reversal interference on all return trajectories in the given system $[16,23,24]$, assuming the spin precessions caused by the Rashba and residual SOIs $[14,28,29]$. The strengths of the SOIs are parametrized by the spin precession angles per unit length, which are twice the SOI-related wave numbers $k_{\alpha} \equiv \frac{m^{*} \alpha}{\hbar^{2}}\left(m^{*}\right.$ and $\hbar$ being the effective mass and Planck constant $h$ divided by $2 \pi$, respectively) and $k_{\mathrm{c}}$ for the Rashba and residual SOIs, respectively, where the explicit correspondence between the SOIs and actual motions of spins was maintained.

The methodology we demonstrate here is applicable to a wide range of nanoscale devices and structures, where sizes and shapes of the nanostructures can be used as a measurement reference. For example, it should be useful in investigating various one-electron interactions $H^{\prime}$ in emerging two-dimensional (2D) systems such as graphene [30,31], transition-metal dichalcogenides [32], and oxide interfaces [33], if nanostructured patterns are introduced to them either naturally or artificially. We note that one-electron interactions $H^{\prime}$ here are not limited to the SOIs. They may also be the Zeeman effect or interactions related to the pseudospin and/or valley degree of freedom of electrons [31].

\section{EXPERIMENT}

\section{A. Fabrication and MC measurement of MRAs}

The lattice-matched $\quad \operatorname{In}_{0.52} \mathrm{Al}_{0.48} \mathrm{As} / \mathrm{In}_{0.53} \mathrm{Ga}_{0.47} \mathrm{As}$ $(10 \mathrm{~nm}) / \mathrm{In}_{0.52} \mathrm{Al}_{0.48} \mathrm{As} \quad \mathrm{QW}$ samples were grown on semi-insulating (001)InP substrate by metal-organic chemical vapor deposition (MOCVD) [Fig. 1(d)]. The Rashba SOI coefficient $\alpha$ was controllable by the external gate voltage from negative to positive values and the Dresselhaus SOI was found to be small $\left(\gamma \lesssim 3.5 \mathrm{eV} \AA^{3}\right)$ in our wafers [14].

Hall bars of the size $125 \times 250 \mu \mathrm{m}^{2}$ were defined by photolithography and wet etching using citric acid $-\mathrm{H}_{2} \mathrm{O}_{2}$ solution. The MRA pattern was fabricated by electron beam lithography with an acceleration voltage of $100 \mathrm{kV}$ and reactive ion etching using the electron cyclotron resonance (ECR) plasma in a $\mathrm{BCl}_{3}$ ambient. The MRA pattern covered the entire Hall bar as in Fig. 1(c). The sample surface was subsequently coated with a 20-nm-thick $\mathrm{HfO}_{2}$ layer for the gate insulation by atomic layer deposition (ALD). Finally, a Au top gate ( $150 \mathrm{~nm}$ thick), which covers the entire Hall bar, was deposited using a 3-nm-thick Ti layer as a wetting layer. The actual devices consisted of 5408 rings $(52 \times 104)$, interconnected with neighboring rings, in a $125 \times 250 \mu \mathrm{m}^{2}$ Hall bar device as in Fig. 1(c). The hard wall potential formed by the reactive ion etching defines the MRA shape [Fig. 1(d)]. The sheet carrier densities $N_{\mathrm{S}}$ and electron mean-free paths $\ell$ of the pristine bulk 2DES varied (a)

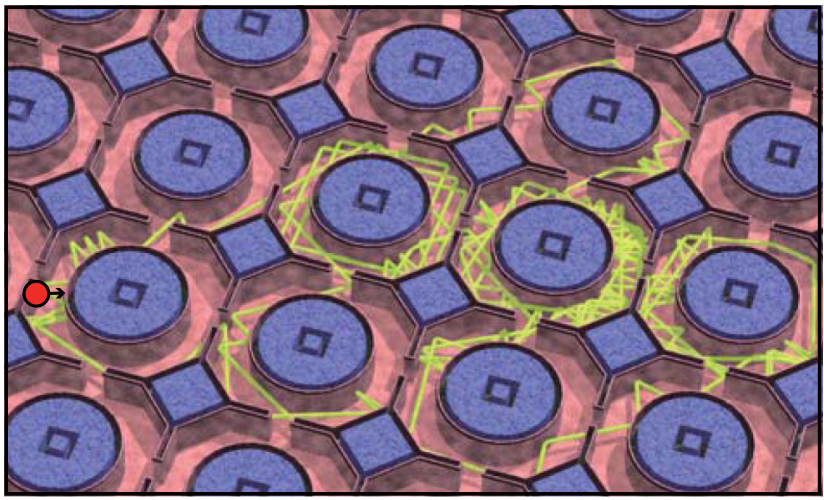

(b)

(c)

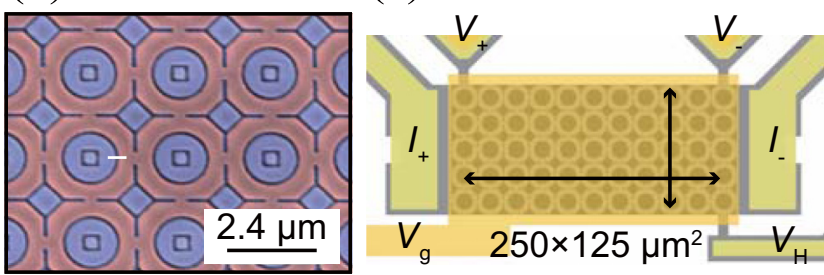

(d)

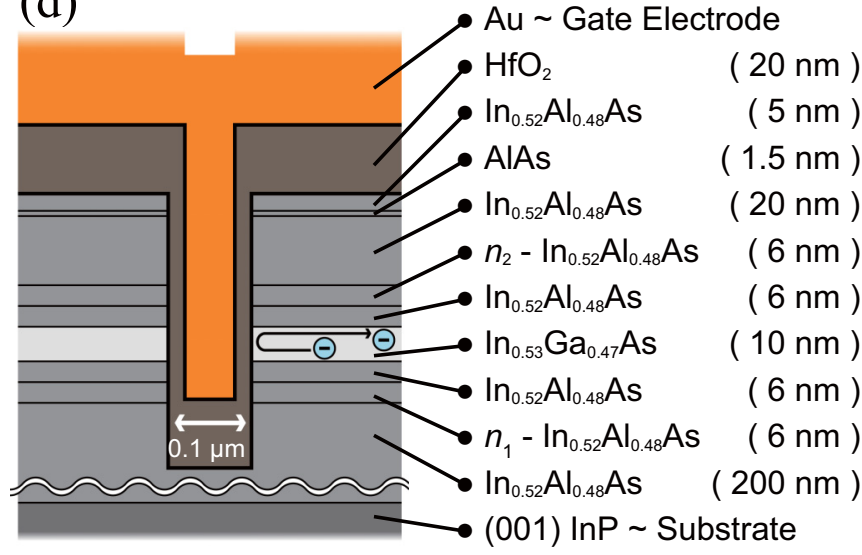

FIG. 1. MRA sample fabricated by nanolithography. (a) An example of chaotic billiard ball trajectory within the MRA that returns to the initial point indicated by red filled circle (CG). (b) Scanning electron micrograph of the fabricated MRA. Colors were added to the original image for clarity. The reddish-colored region contributes to the electron transport, whereas the bluish-colored regions are isolated areas that do not contribute to the transport. The dark lines and curves are the ditches created by reactive ion etching, where squares drawn inside the circles are just to adjust the proximity effect in the electron beam lithography. (c) Illustration showing the arrangement of the MRA in a Hall bar device. (d) Cross-sectional illustration of the MRA device along the white line segment indicated in (b) and the MOCVD-grown epitaxial layer structure. The doping densities $\left(n_{1}, n_{2}\right)$ are $\left(1.5 \times 10^{24} \mathrm{~m}^{-3}, 2.5 \times 10^{24} \mathrm{~m}^{-3}\right)$ and $\left(2.0 \times 10^{24} \mathrm{~m}^{-3}\right.$, $2.0 \times 10^{24} \mathrm{~m}^{-3}$ ) for the first (Sec. IIB) and second (Sec. IVD) samples in the main text, whose sample names are KH3-1 and KH1-3, respectively.

from $1.3 \times 10^{16}$ to $2.4 \times 10^{16} \mathrm{~m}^{-2}$ and from 1.1 to $2.2 \mu \mathrm{m}$, respectively, for gate voltages from 0.2 to $1.4 \mathrm{~V}[16,34,35]$.

Figures 1(a) and 1(b) depict the shape and size of our MRA structure, where Fig. 1(a) also shows an example of 


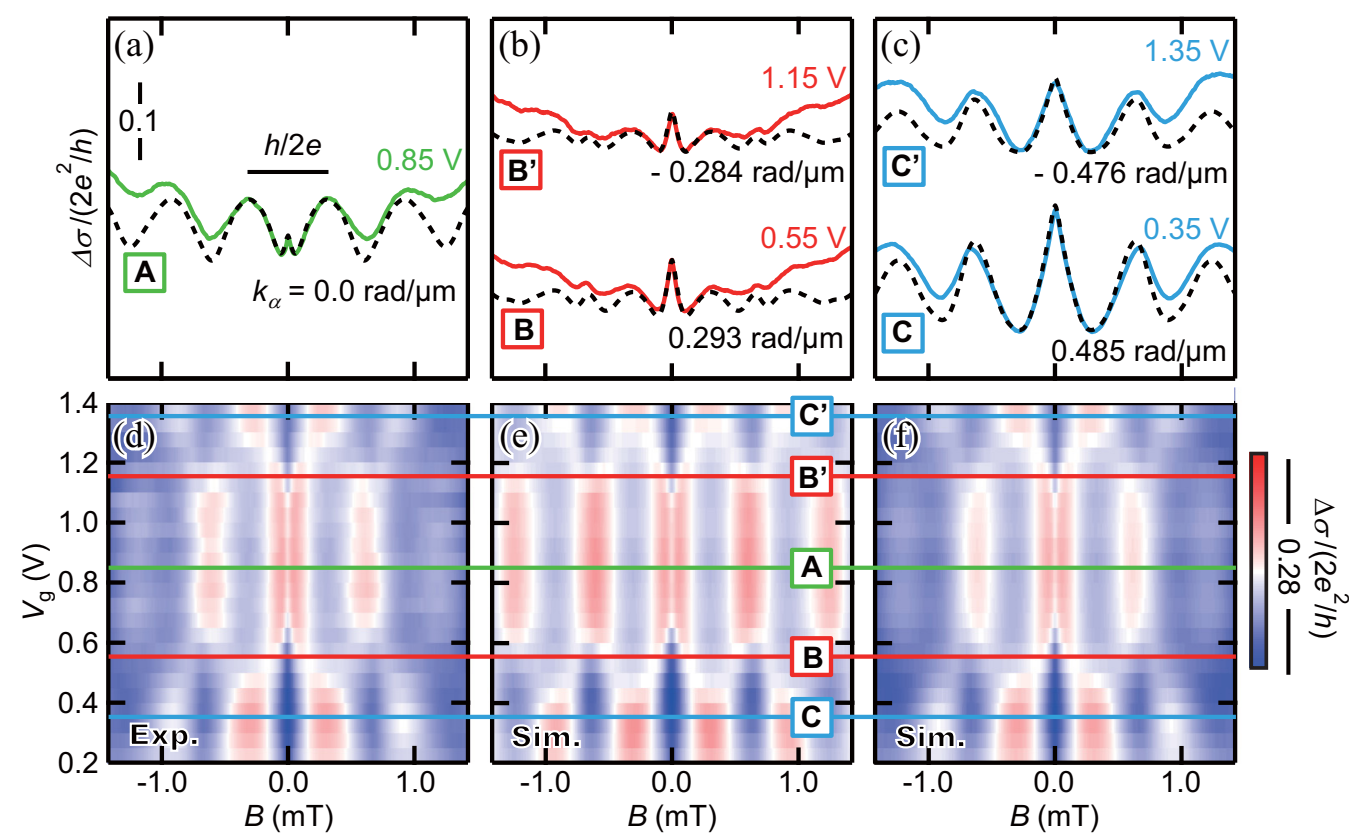

FIG. 2. Gate dependence of the MC curves of MRA. (a)-(c) (Colored curves) Experimental MC curves $(\Delta \sigma)$ at selected gate voltages $\left(V_{\mathrm{g}}\right)$ corresponding to the horizontal lines $A-C, B^{\prime}$, and $C^{\prime}$ in (d). (Black dashed curves) $\Delta \sigma$ generated by the "chaos only" model [see (e)] maximizing the $r^{2}$ values with the corresponding experimental MC curves (see Sec. IIID). (d)-(f) Image plots of $\Delta \sigma$ from (d) the experiment at a dilution refrigeration temperature, (e) the "chaos only" model, and (f) the "chaos+impurity" model. $k_{\alpha}$ values in (a)-(c), (e), and (f) are related to $V_{\mathrm{g}}$ by $k_{\alpha}=-0.9614 \times\left(V_{\mathrm{g}}-0.8547\right)(\mathrm{rad} / \mu \mathrm{m})$.

chaotic return trajectories within the MRA (see S1 in the Supplemental Material [36] for more detailed information). The convex curvature introduced in the MRA shape, as in Fig. 1(b), makes the trajectories inherently chaotic. This is an important improvement from our previous studies $[11,12,16]$.

The MC measurements of the MRA samples were performed using the standard ac lock-in method in a dilution refrigerator at temperatures below $1 \mathrm{~K}$. The excitation voltages in the measurement were so controlled that the excited energies were well below the thermal energy $k_{\mathrm{B}} T$.

\section{B. Gate dependence of MC curves in MRA}

Shown in Figs. 2(a)-2(c) (colored curves) are examples of experimental MC curves with several different $V_{\mathrm{g}}$ measured at the base temperature of the dilution refrigerator $(T=15 \mathrm{mK}$ at the mixing chamber). The dashed curves in these figures represent the simulation results of the "chaos only" model as discussed in Sec. III. The electron temperature $T_{\mathrm{el}}$ [37] in these measurements was $\sim 100 \mathrm{mK}$ (S2 in the Supplemental Material [36] and Sec. IV D). We define the oscillating component of the MC curve as $\Delta \sigma(B)=\sigma(B)-\sigma_{0.16 \mathrm{mT}}$ at each $V_{\mathrm{g}}$, where $\sigma_{0.16 \mathrm{mT}}$ is the conductivity value at $B=0.16 \mathrm{mT}$. This $B$ value was chosen to be at $\frac{1}{4}$ of the primary $h / 2 e$ oscillation period (phase $\pi / 2$ ) as the amplitude of the main oscillation (cosine wave) becomes zero $[ \pm \cos (\pi / 2)=0]$, where $h$ and $e$ are Planck's constant and elementary charge, respectively.

The observed $\Delta \sigma(B)$ reflected the AAS-type quantum interference and the WL/WAL effect, as expected. We recognize the AAS-type oscillations clearly in the data with $V_{\mathrm{g}}=0.35,0.85$, and $1.35 \mathrm{~V}$ [Figs. 2(a) and 2(c)]. The period of oscillation turned out to be $\Delta B=h /\left(2 e S_{0}\right)=0.64 \mathrm{mT}$, where
$S_{0}=1.8 \times 1.8 \mu \mathrm{m}^{2}$ is the typical encircling area for a closed loop within a unit ring of the MRA. The negative MC effect around $B=0 \mathrm{mT}$ in Fig. 2(c) is the manifestation of the strong Rashba SOI induced by $\left\langle E_{z}\right\rangle$. In contrast, the primary $h / 2 e$ oscillation of $\Delta \sigma(B)$ appears convex downward around $B=0 \mathrm{mT}$ for $V_{\mathrm{g}}=0.85 \mathrm{~V}$ [Fig. 2(a)], where the Rashba SOI is minimized by realizing $\left\langle E_{z}\right\rangle=0 \mathrm{~V} / \mathrm{m}$. A small and sharp peak (WAL peak) is still noticeable around $B=0 \mathrm{mT}$ in Fig. 2(a). This is due to a relatively long phase coherent length of electrons (realized by $T_{\mathrm{el}} \approx 100 \mathrm{mK}$ ) and finite residual SOI $[14,28,29]$. The latter should not be attributed to the Dresselhaus SOI automatically because the Dresselhaus SOI was negligible relative to the Rashba SOI in our particular InGaAs/InAlAs QWs that are lattice matched to (001) InP [14]. While physical phenomena related to the interplay between the Rashba and Dresselhaus effects [38-40] are not a focus in this work for this reason, the simulation analysis presented here is still useful for such studies if relevant experimental data are available from other material systems.

The data in Fig. 2(b) $\left(V_{\mathrm{g}}=0.55\right.$ and $\left.1.15 \mathrm{~V}\right)$ represent the cases where the primary $h / 2 e$ oscillation is diminished substantially by carefully adjusting the SOI strength by the gate voltage $[11,12,16]$. This adjustment was made by setting the spin precessions per side of polygonal closed loops to some special angles [41]. For example, such angles are given to be $(1 \pm 0.576) \pi$ and $(1 \pm 0.178) \pi$ in a square loop considering only the Rashba SOI [11]. The corresponding angle in this work was found to be $2 k_{\alpha} L \approx 0.33 \pi$ rad using the side length $L=1.8 \mu \mathrm{m}$ and $k_{\alpha}=0.29 \mathrm{rad} / \mu \mathrm{m}$ (see Sec. IV A), which is in qualitative agreement with the smallest found in the simple square-loop model $(0.424 \pi)$ [11]. An equivalent physical phenomenon was predicted for open quantum dots as 
well [8]. As a result, the WAL peaks in Fig. 2(b) are composed exclusively of the higher harmonic oscillations $(h / 2 n e$ with $n \geqslant 2)$.

\section{Onsager symmetry relevant to $\left\langle E_{z}\right\rangle$}

The image plot of the experimental MC data, setting the abscissa and ordinate as $B$ and $V_{\mathrm{g}}$, respectively, is displayed in Fig. 2(d) to show how the MC curves evolve with $V_{\mathrm{g}}$. In this plot, we clearly see the phase flips in the primary $h / 2 e \mathrm{MC}$ oscillations across the horizontal lines $B$ and $B^{\prime}$ as we vary $V_{\mathrm{g}}$. These boundaries ( $B$ and $B^{\prime}$ ) correspond to the special spin precession angles that diminish the $h / 2 e$ oscillation, as discussed in the preceding section. The WL-to-WAL-like transition of the AAS effect was more traditionally observed by the introduction of strong SOI scatterers $[42,43]$. The similar phenomenon observed here, on the other hand, should be regarded as an evidence of coherent spin precession by the Rashba SOI.

We notice that Fig. 2(d) is roughly symmetric about the horizontal line $A$ at $V_{\mathrm{g}}=0.85 \mathrm{~V}$ in addition to the more commonly known symmetry about the vertical line at $B=0 \mathrm{mT}$. Both symmetries are results of the Onsager reciprocity [44] or a generalized version of it. While the latter is known as the regular Onsager relation, i.e., $\Delta \sigma\left(\left\langle E_{z}\right\rangle, B\right)=\Delta \sigma\left(\left\langle E_{z}\right\rangle,-B\right)$, the former, associated with flipping the relativistic magnetic field $\mathbf{B}_{\text {SOI }}(\mathbf{k})$, i.e., $\Delta \sigma\left(\left\langle E_{z}\right\rangle, B\right) \approx \Delta \sigma\left(-\left\langle E_{z}\right\rangle, B\right)$, is an observation that has never been reported elsewhere in mesoscopic ring systems.

The observation of the approximate symmetry $\Delta \sigma\left(\left\langle E_{z}\right\rangle, B\right) \approx \Delta \sigma\left(-\left\langle E_{z}\right\rangle, B\right)$ is surprising considering the highly gate-dependent conditions in our experiment, such as the carrier density, mobility, gate depletion, and the effective impurity arrangement within the MRA. We attribute the robustness of the $\Delta \sigma\left(\left\langle E_{z}\right\rangle, B\right) \approx \Delta \sigma\left(-\left\langle E_{z}\right\rangle, B\right)$ relation to the universal nature of chaos. This means that $\Delta \sigma$ is rather unaffected by specific details of the electron trajectories. We conclude that $\left\langle E_{z}\right\rangle=0 \mathrm{~V} / \mathrm{m}$ is realized at $V_{\mathrm{g}}=0.85 \mathrm{~V}$ from the observation of this symmetry.

\section{SEMICLASSICAL BILLIARD BALL SIMULATION}

\section{A. Justification of the present model}

The observation of the variation of the Onsager relation $\Delta \sigma\left(\left\langle E_{z}\right\rangle, B\right) \approx \Delta \sigma\left(-\left\langle E_{z}\right\rangle, B\right)$ urged us to develop a semiclassical billiard ball simulation $[1,3,5,8,16,23,24]$ on our MRA system to understand the experimental results more quantitatively. The main improvements in the present model, relative to our previous ones $[11,12,16]$, include the following: (1) The MRA shape in this work provides chaotic billiards inherently, whereas the trajectories in the previous MRA shape [16] are categorized to be "regular" (integrable), which is not ideal in the framework of a semiclassical approximation. (2) The coordinate points at which we start billiards in this work are homogeneously distributed over the conductive region of the MRA, the reddish-colored region in Fig. 1(b) (see S1 in the Supplemental Material [36] for more information). (3) The precise definition of "return" for billiard ball trajectories is fixed in such a way that quantitative values of $\Delta \sigma$ can be obtained directly from the simulation.
We adopted the real-space approach (Boltzmannian picture in Ref. [45]), which turned out to be exact in describing the diffusive WL/WAL effect of a quasi-2DES [23,24]. The validity of this approach in our semiballistic MRA system is conjectured from the accumulated knowledge in the quantum chaos research [1-5]. A series of successful results in this work can be regarded as an elaborate experimental proof of this conjecture.

In practicing the idea, the processes of scattering are simplified to two of the followings in our MRA: (1) specular reflection of electrons by the hard wall boundaries that define the MRA shape and (2) isotropic probabilistic scattering at a rate of $d l / \ell_{\mathrm{ex}}$ for an infinitesimal length of electron passage $d l$ with an extrinsic scattering length $\ell_{\mathrm{ex}}$. It can be understood that the latter, primarily representing the impurity scattering in the pristine 2DES (but the value is somewhat reduced by the fabrication process), is considered additionally to the former (scattering from geometric features [1]). It turned out that these two processes are required minimally for reproducing the experimental MC curves quantitatively in our MRAs. It can be said that this bold simplification was made possible by the fact that the actual MRA shape sensed by the 2D electrons was not much altered by the external gate voltage (or gate depletion) at least for the given $V_{\mathrm{g}}$ range.

\section{B. Details of billiard ball simulation}

In the adopted semiclassical approximation, the propagation amplitude of an electron, that is, the path-integral representation of the Feynman propagator, is replaced by a summation over classical paths. These classical paths may be considered as a random walk. We let a billiard ball (electron) start at some point $\mathbf{r}_{1}$ within the conductive region of MRA [Fig. 1(b) and S1 in the Supplemental Material [36]] with some azimuthal angle $\theta_{1}$ and generate closed loops associated with this initial condition multiple times whenever the billiard ball returns to $\mathbf{r}_{1}$. In generating these closed loops, a cutoff $L_{\max }$ is introduced for the total ballistic length of the billiard trajectories. The definition of return is that the electron passes by the initial point $\mathbf{r}_{1}$ more closely than some predetermined distance $\Delta r$. For example, if the first closed loop associated with the given initial condition $\left(\mathbf{r}_{1}, \theta_{1}\right)$ is $\mathbf{r}_{1} \rightarrow \mathbf{r}_{2} \rightarrow \cdots \rightarrow \mathbf{r}_{N_{1}} \rightarrow \mathbf{r}_{1}$, the second one with the same initial condition may be $\mathbf{r}_{1} \rightarrow \mathbf{r}_{2} \rightarrow$ $\cdots \rightarrow \mathbf{r}_{N_{1}} \rightarrow \mathbf{r}_{N_{1}+1} \rightarrow \cdots \rightarrow \mathbf{r}_{N_{2}} \rightarrow \mathbf{r}_{1}$, where the distance between the line segment $\mathbf{r}_{N_{1}} \rightarrow \mathbf{r}_{N_{1}+1}$ and the point $\mathbf{r}_{1}$ is smaller than $\Delta r$. Scattering takes place both deterministically (from the geometric feature) and probabilistically (according to $\left.d l / \ell_{\mathrm{ex}}\right)$ using a pseudo-random-number generator [24]. The number of closed loops generated from a single initial condition seldom exceeded 10 for $L_{\max }=3000 \mu \mathrm{m}$. The number of all closed loops generated in this way, from all initial conditions, was confirmed to be proportional to the value of $\Delta r$ chosen, provided $\Delta r$ is sufficiently smaller than a typical distance between the collisions $(\sim 0.5 \mu \mathrm{m}$ in our case). We chose $\Delta r=0.05 \mu \mathrm{m}$ in this study to maximize the efficiency and accuracy of the calculation simultaneously. The number of all initial conditions used was 244224 (S1 in the Supplemental Material [36]). The numbers of all closed loops generated ranged from 196228 for $\ell_{\mathrm{ex}}=10 \mu \mathrm{m}$ to 309461 for $\ell_{\mathrm{ex}}=1 \mu \mathrm{m}\left(L_{\max }=3000 \mu \mathrm{m}\right)$. 
Next, we define the nominal return count density $W_{0}\left(\ell_{\mathrm{ex}}, L_{\max }\right)$ by (number of all closed loops)/(number of all initial conditions $\times 2 \Delta r$ ). For $L_{\max }=3000 \mu \mathrm{m}$, we found $W_{0}=12.67$ and $8.035 \mu \mathrm{m}^{-1}$ for $\ell_{\mathrm{ex}}=1$ and $10 \mu \mathrm{m}$, respectively. The modified return count density $W\left(B, k_{\alpha}, k_{\mathrm{c}}, \ell_{\mathrm{ex}}, L_{\phi}\right)$ is defined as follows using $i$ to index all the closed loops generated:

$$
W\left(B, k_{\alpha}, k_{\mathrm{c}}, \ell_{\mathrm{ex}}, L_{\phi}\right)=\frac{\sum_{i}\left\{A_{i}\left(k_{\alpha}, k_{\mathrm{c}}\right) \times \cos \left(2 e B S_{i} / \hbar\right) \times\left(1+\cos \theta_{i}\right) \times \exp \left(-L_{i} / L_{\phi}\right)\right\}}{(\text { number of all initial conditions }) \times(2 \Delta r)},
$$

where $S_{i}, L_{i}$ and $\theta_{i}$ are the encircling area, total length of the billiard ball trajectory, and difference between the initial and final (returning) azimuthal angles, associated with the $i$ th closed loop, respectively. Various quantum-mechanical modifications to the simple Boltzmannian picture [45] are $A_{i}\left(k_{\alpha}, k_{\mathrm{c}}\right)$, the Aharonov-Casher correction due to spin precession [11-13] $\left(k_{\alpha}=\frac{m^{*} \alpha}{\hbar^{2}}\right.$ and $k_{c}$ being halves of the spin precession angles per unit length by the Rashba and residual SOIs, respectively); $\cos \left(2 e B S_{i} / \hbar\right)$, the Aharonov-Bohm correction due to the threading magnetic flux; $\left(1+\cos \theta_{i}\right)$, non-back-scattering correction for the billiard loop [23,46]; and $\exp \left(-L_{i} / L_{\phi}\right)$, phenomenological prescription of electron phase decoherence, respectively. $A_{i}\left(k_{\alpha}, k_{\mathrm{c}}\right)$ is given by a half of the trace of $\mathbf{R}_{\text {tot }}^{2}[16,24]$, letting $\mathbf{R}_{\text {tot }}$ be the total spin rotation operator by the SOIs for a given closed loop (in either the clockwise or counterclockwise direction). We note that $A_{i}\left(k_{\alpha}, k_{\mathrm{c}}\right)$ is real and $-1 \leqslant A_{i}\left(k_{\alpha}, k_{\mathrm{c}}\right) \leqslant 1$. We also note $\exp \left(-L_{i} / L_{\phi}\right)=\exp \left(-t_{i} / \tau_{\phi}\right)$, letting $t_{i} \equiv L_{i} / v_{\mathrm{F}}$. Thus, our $L_{\phi}$ differs from the traditional diffusive decoherence length $\ell_{\phi}=\sqrt{D \tau_{\phi}}$.

One may be concerned whether our experiment/analysis is in ballistic or diffusive regime. The dissipative damping usually depends on time as $\exp \left(-t / \tau_{\phi}\right)$ without assuming ballistic or diffusive regime. Then, $\tau_{\phi} \ll \tau_{e}$ corresponds to ballistic transport where $\ell_{\phi}=L_{\phi}=v_{\mathrm{F}} \tau_{\phi}$. On the other hand, $\tau_{\phi} \gg \tau_{e}$ corresponds to diffusive transport where $\ell_{\phi}=\sqrt{D \tau_{\phi}}$. Within this standard picture, the diffusive phase coherence length $\ell_{\phi}$ is given by $\sqrt{L_{\phi} \ell / 2} \approx 10 \mu \mathrm{m}$, where $\ell$ is the mean-free path. The bottom line is that one does not have to assume diffusive/ballistic regimes a priori $[47,48]$.

We can convert $W$ to the quantum correction term to the electrical conductivity $\Delta \sigma_{\text {sim }}$ using the following equation that is proven to be correct for the WL/WAL effect in a quasi-2DES [23-27]:

$$
\frac{\Delta \sigma_{\text {sim }}(B)}{\sigma_{0}}=-\frac{\lambda_{\mathrm{F}}}{\pi} \times W\left(B, k_{\alpha}, k_{\mathrm{c}}, \ell_{\mathrm{ex}}, L_{\phi}\right),
$$

where $\lambda_{\mathrm{F}}$ is the Fermi wavelength and $\sigma_{0}$ is the electrical conductivity of the MRA in the classical limit. $\sigma_{0}$ is assumed to be constant in the given range of the magnetic field, where $\omega_{\mathrm{c}} \tau \ll 1$ ( $\omega_{\mathrm{c}}$ is the cyclotron frequency).

\section{C. "Chaos only" and "chaos+impurity" models}

The first simulation, denoted as the "chaos only" model, was performed considering only the chaotic trajectories inherent to the MRA shape, where a sufficiently large value of $10 \mu \mathrm{m}$ was used for $\ell_{\mathrm{ex}}$. It turned out that the introduction of $\ell_{\mathrm{ex}}$ was necessary for eliminating "regular" (integrable) trajectories completely. However, the value of $\ell_{\mathrm{ex}}$ could be chosen rather arbitrarily as far as $\ell(\approx 1 \mu \mathrm{m}) \ll \ell_{\mathrm{ex}} \ll L_{\phi}$, where $\ell$ is the mean-free path in the pristine $2 \mathrm{DES}$. The second simula- tion, denoted as the "chaos+impurity" model, was performed using relatively small values of $\ell_{\mathrm{ex}}$ (approximately $1 \mu \mathrm{m}$ ), treating it as a fitting parameter. In both simulations, the strength of the Rashba SOI was incorporated as the spin precession angle per unit length, given by $2 k_{\alpha}(\mathrm{rad} / \mu \mathrm{m})$, where $k_{\alpha} \equiv m^{*} \alpha / \hbar^{2}$ in the pristine bulk 2DES [11]. Similarly, we denote the spin precession angle per unit length due to the residual SOI by $2 k_{\mathrm{c}}(\mathrm{rad} / \mu \mathrm{m})$ [14]. The residual SOI was emulated using the cubic Dresselhaus Hamiltonian $H_{\mathrm{D} \pm}^{(3)}=$ $\pm \frac{\gamma}{4} k^{3}\left\{\sigma_{x} \cos (3 \theta)+\sigma_{y} \sin (3 \theta)\right\}$ (double sign in same order) employing the following symmetrization procedure, although we reiterate that the origin of it can not be the Dresselhaus SOI automatically (Sec. IIB). Namely, the symmetrized $\Delta \sigma(B)$ was given by the average of $\Delta \sigma(B)$ s between one obtained with a pair of Hamiltonians $\left(H_{\mathrm{R}}, H_{\mathrm{D}+}^{(3)}\right)$ and the other obtained with the other pair $\left(H_{\mathrm{R}}, H_{\mathrm{D}-}^{(3)}\right)$. This procedure is to eliminate a particular symmetry hidden in these Hamiltonians when they are combined $\left(H^{\prime}=H_{\mathrm{R}}+H_{\mathrm{D} \pm}^{(3)}\right)$ [24]. In any case, our experiment was unable to resolve the difference between these two possible combinations of the Hamiltonians, i.e., $\left(H_{\mathrm{R}}, H_{\mathrm{D}+}^{(3)}\right)$ or $\left(H_{\mathrm{R}}, H_{\mathrm{D}-}^{(3)}\right)$.

Given a direction of the electron passage by a unit vector $(\cos \theta, \sin \theta)$, the spin precession axes associated with $H_{\mathrm{R}}$ and $H_{\mathrm{D} \pm}^{(3)}$ are given by unit vectors $(\sin \theta,-\cos \theta)$ and $( \pm \cos 3 \theta, \pm$ $\sin 3 \theta$ ), respectively (double sign in same order). It is reminded that the corresponding spin precession angles per unit length are given by $2 k_{\alpha}$ and $2 k_{c}$, respectively. The value of the cutoff length $\left(L_{\max }=3000 \mu \mathrm{m}\right)$ used in this work is large enough to cover the phase coherent length $L_{\phi}=v_{\mathrm{F}} \tau_{\phi}$ up to $500 \mu \mathrm{m}$ considering $\exp \left(-\frac{L_{\max }}{L_{\phi}}\right)=2.5 \times 10^{-3} \ll 1$ for $L_{\max } / L_{\phi}=6$, where $v_{\mathrm{F}}$ and $\tau_{\phi}$ are the Fermi velocity and phase coherent time, respectively.

\section{Extraction of parameters by maximizing $\boldsymbol{r}^{2}$}

The extraction of the parameters in the billiard ball simulation was performed by maximizing the value of the coefficient of determination $r^{2}$ between the experimental and simulated $\Delta \sigma(B)$ s for given ranges of the magnetic field, ruling out the cases of negative correlation between them. The definition of $r^{2}$ we used is

$$
r^{2}=\frac{\left\{\sum_{i}\left(x_{i}-\bar{x}_{i}\right) \cdot\left(y_{i}-\bar{y}_{i}\right)\right\}^{2}}{\sum_{i}\left(x_{i}-\bar{x}_{i}\right)^{2} \cdot\left(y_{i}-\bar{y}_{i}\right)^{2}},
$$

where $\left\{x_{i}\right\}$ and $\left\{y_{i}\right\}$ are the sets of data to be compared to each other. The maximization of the $r^{2}$ value can be done independently of the value of $\lambda_{\mathrm{F}}$ in Eq. (2). Therefore, the value of $\lambda_{\mathrm{F}} \sigma_{0} / \pi$ was obtained deterministically by equating the variances of the experimental and theoretical $\Delta \sigma(B) \mathrm{s}$ after optimizing the other parameters $k_{\alpha}, k_{\mathrm{c}}, \ell_{\mathrm{ex}}$, and $L_{\phi}$. A rational 
(a)

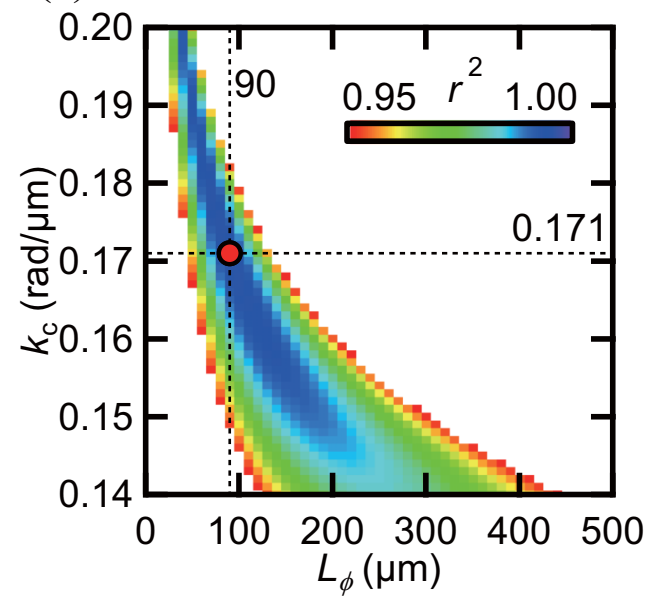

(b)

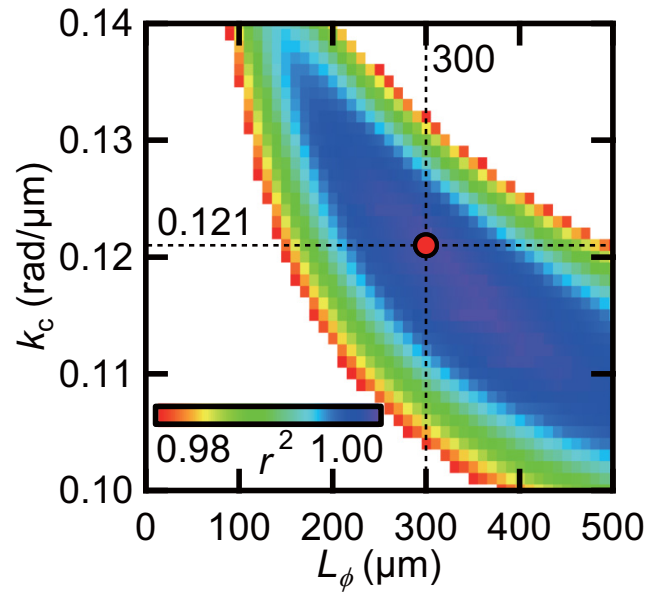

FIG. 3. $r^{2}$ values (see Sec. IIID) calculated between the measured and simulated MC curves at $V_{\mathrm{g}}=0.85 \mathrm{~V}$, where $k_{\alpha}=0 \mathrm{rad} / \mu \mathrm{m}$, as a function of $k_{\mathrm{c}}$ and $L$. The magnetic field ranges used in the calculation of $r^{2}$ are $|B| \leqslant 0.32 \mathrm{mT}$ and $|B| \leqslant 1.42 \mathrm{mT}$ in the "chaos only" and "chaos+impurity" models, respectively. (a) Result in the chaos only model $\left(\ell_{\mathrm{ex}}=10 \mu \mathrm{m}\right)$. (b) Result in the chaos+impurity model $\left(\ell_{\mathrm{ex}}=1 \mu \mathrm{m}\right)$. Only the regions corresponding to $r^{2} \geqslant 0.95$ and $r^{2} \geqslant 0.98$ with positive correlation are color scaled in (a) and (b), respectively.

approach to extract these parameter values is discussed in the next section.

\section{RESULTS AND DISCUSSION}

Because of the vast $R^{4}$ parameter space $\left(k_{\alpha}, k_{\mathrm{c}}, \ell_{\mathrm{ex}}\right.$, and $\left.L_{\phi}\right)$ to be explored for a unique theoretical $\Delta \sigma(B)$ curve, one should make the maximum use of physical insight in searching the right sets of the parameter values efficiently. We present below the final refined version of our procedure, after an appreciable number of trials and errors, where cross-checks of the results among experiment, "chaos only" model and "chaos+impurity" model, were routinely made.

\section{A. Extraction of the $k_{\alpha}$ vs $V_{\mathrm{g}}$ relation by "chaos only" model}

In our most successful procedure of parameter extraction, we first extracted the $k_{\alpha}$ vs $V_{\mathrm{g}}$ relation using the chaos only model, where the linearity between $k_{\alpha}$ and $V_{\mathrm{g}}$ is assumed based on the experimentally verified relation $\alpha \propto\left\langle E_{z}\right\rangle$ [14]. As a prerequisite for this extraction, the experimental $\Delta \sigma(B)$ at $V_{\mathrm{g}}=0.85 \mathrm{~V}$, where $k_{\alpha}=0$ is realized, was analyzed with only two parameters $k_{\mathrm{c}}$ and $L_{\phi}$. Here, we obtained $k_{\mathrm{c}}=$ $0.171 \mathrm{rad} / \mu \mathrm{m}$ and $L_{\phi}=90 \mu \mathrm{m}$ as in Fig. 3(a). Fixing these values temporarily, we extracted the $k_{\alpha}$ vs $V_{\mathrm{g}}$ relation first. A naive justification of this approach is that $k_{\mathrm{c}}$ and $L_{\phi}$ are not dependent on $V_{\mathrm{g}}$ explicitly, whereas $k_{\alpha}$ has a more direct dependence on $V_{\mathrm{g}}$. We await more quantitative values of $k_{\mathrm{c}}$ and $L_{\phi}$ until the next subsection, where the chaos+impurity model is discussed.

In extracting the $k_{\alpha}$ vs $V_{\mathrm{g}}$ relation, an equation $k_{\alpha}=-a \times$ $\left(V_{\mathrm{g}}-b\right)$ was assumed and the values of $a$ and $b$ were determined in such a way that the sum of the $r^{2}$ values for all 25 pairs of theoretical and experimental $\Delta \sigma(B) \mathrm{s}$ in the gate-voltage range $0.2 \mathrm{~V} \leqslant V_{\mathrm{g}} \leqslant 1.4 \mathrm{~V}$ becomes maximum. The magnetic field range used in this procedure was $|B| \leqslant 0.32 \mathrm{mT}$, which corresponds to a single period of $h / 2 e$ oscillation. This is because the chaos only model cannot reproduce the envelope part of the MC oscillation as in Fig. 5. Here, we obtained $a=0.9614 \mathrm{rad} / \mu \mathrm{m} \mathrm{V}$ and $b=0.8547 \mathrm{~V}$. These values are translated to the $k_{\alpha}$ values of 0.524 and $-0.629 \mathrm{rad} / \mu \mathrm{m}$ at $V_{\mathrm{g}}=0.2$ and $1.4 \mathrm{~V}$, respectively. The reason for the narrowness of the range of $B$ used here $(|B| \leqslant 0.32 \mathrm{mT})$ is explained as follows. A sensitive feature in the chaos only model turned out to be the WAL feature observed around $B=0 \mathrm{mT}$ in $\Delta \sigma(B)$. Theoretically, this WAL feature is most largely affected by the closed loops originating from the inherent chaotic trajectories, which encircle relatively large areas, hence the narrowness of the range in $B$ used in the analysis.

The temporal usage of the parameter values for $k_{\mathrm{c}}$ and $L_{\phi}$ was also justified by observing the stability of the resultant values of $a$ and $b$ against some changes in the values of $k_{\mathrm{c}}$ and $L_{\phi}$. The upper limit (bound) for the possible value of $k_{\mathrm{c}}$ is $0.29 \mathrm{rad} / \mu \mathrm{m}$ because this is the value of $\left|k_{\alpha}\right|$ around which the primary $h / 2 e$ oscillation was diminished substantially as in Fig. 2(b), but the effect from $k_{c}$ is not visible. We note that the total spin precession angle can be understood as a kind of vector sum between $k_{\mathrm{c}}$ and $k_{\alpha}$. Therefore, most MC curves are governed by either of the two parameters, $k_{\mathrm{c}}$ or $k_{\alpha}$, whichever larger. The image plot of the MC curves obtained by the chaos only model is given in Fig. 2(e).

\section{B. Extraction of parameters by "chaos+impurity" model}

We define the questions to be answered in the "chaos+impurity" model as follows: (1) Can we confirm the $k_{\alpha}$ vs $V_{\mathrm{g}}$ relation that we obtained in the chaos only model? (2) Can we refine the temporal values of $k_{\mathrm{c}}$ and $L_{\phi}$ and extract new parameter values $\ell_{\mathrm{ex}}$ and $\lambda_{\mathrm{F}}$ properly?

To answer these questions, we first refitted the data at $V_{\mathrm{g}}=0.85 \mathrm{~V}\left(k_{\alpha}=0 \mathrm{rad} / \mu \mathrm{m}\right)$ using only $k_{\mathrm{c}}$ and $L_{\phi}$ as free fitting parameters in the magnetic field range $|B| \leqslant 0.32 \mathrm{mT}$, but now assuming $\ell_{\mathrm{ex}}=1 \mu \mathrm{m}$ instead of $10 \mu \mathrm{m}$ as used in the chaos only model. The primary purpose of this first procedure is to obtain a reasonable value of $k_{\mathrm{c}}$, where $\ell_{\mathrm{ex}}=1 \mu \mathrm{m}$ was chosen rather arbitrarily from the bulk transport information 

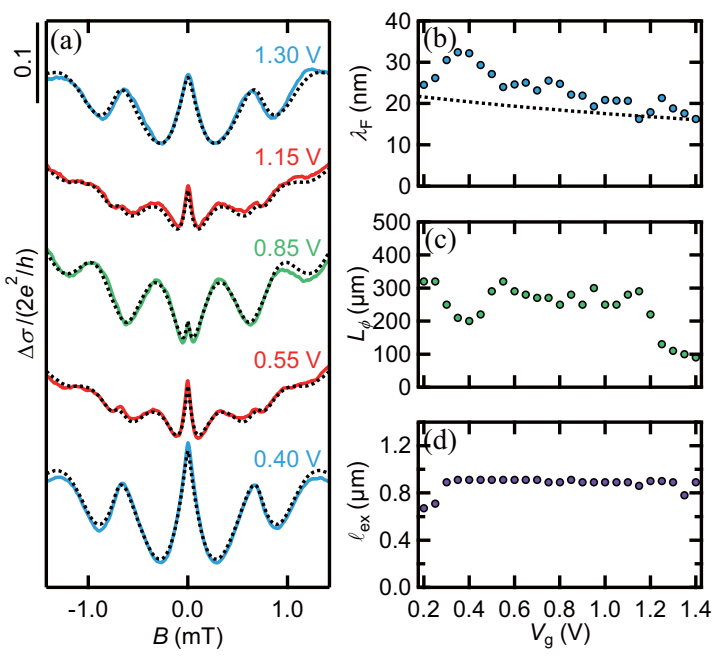

FIG. 4. Parameter extraction from the "chaos+impurity" model. (a) (Colored curves) Replot of the experimental $\Delta \sigma \mathrm{s}$ shown in Figs. 2(a)-2(c). Each curve was shifted vertically for clarity. (Black dotted curves) $\Delta \sigma \mathrm{s}$ generated by the chaos+impurity model maximizing the $r^{2}$ values with the corresponding experimental MC curves. (b)-(d) Various parameter values extracted from the chaos+impurity model as a function the gate voltage $V_{\mathrm{g}}$. These parameters are (b) the Fermi wavelength $\lambda_{\mathrm{F}}$, (c) electron phase coherent length $L_{\phi}$, and (d) extrinsic scattering length $\ell_{\mathrm{ex}}$. The dotted curve in (b) was obtained from Eq. (4) for the pristine bulk 2DES using the $k_{\alpha}$ vs $V_{\mathrm{g}}$ relation extracted in Sec. IV A.

$(0.1 \mu \mathrm{m} \ll \ell \ll 10 \mu \mathrm{m})[16,34,35]$. The extracted $k_{\mathrm{c}}$ value was almost unaffected by small variations of $\ell_{\mathrm{ex}}$ around $1 \mu \mathrm{m}$, which justifies our rather $a d$ hoc approach chosen here. As a result, we obtained $k_{\mathrm{c}}=0.121 \mathrm{rad} / \mu \mathrm{m}$ and $L_{\phi}=300 \mu \mathrm{m}$ from the MC curve at $V_{\mathrm{g}}=0.85 \mathrm{~V}$ as in Fig. 3(b). Because $k_{\mathrm{c}}$ has a dominant role in the MC curves only near $V_{\mathrm{g}}=0.85 \mathrm{~V}$, where the values of $k_{\alpha}$ are smaller than $k_{\mathrm{c}}$, we terminate the investigation of the $k_{\mathrm{c}}$ value here and use this value for fitting the rest of the MC curves as well. The $k_{\mathrm{c}}$ value obtained here is apparently converted to the bulk Dresselhaus parameter $\gamma=6.57 \mathrm{eV} \AA^{3}$ assuming the Dresselhaus Hamiltonian $H_{\mathrm{D} \pm}^{(3)}$. This value is slightly larger than our previous estimate $\gamma<$ $3.5 \mathrm{eV} \AA^{3}$ [14], but more consistent with the result of a thinner QW [40]. We also note the controversial argument about the value of $\gamma$ in general III-V materials against the previously believed one $\left(\gamma \sim 25 \mathrm{eV}^{3}\right)$ [49].

All 25 experimental data with different $V_{\mathrm{g}}$ 's were then fitted with $\ell_{\text {ex }}$ and $L_{\phi}$ only, employing the $k_{\alpha}$ vs $V_{\mathrm{g}}$ relation obtained in the chaos only model. The range of the magnetic field considered here was $|B| \leqslant 1.42 \mathrm{mT}$, which is the maximally available range in the measurements at the base dilution refrigeration temperature $\left(T_{\mathrm{el}} \approx 100 \mathrm{mK}\right)$. The image plot of the MC curves obtained in the chaos+impurity model is displayed in Fig. 2(f), which shows precise agreement with the experimental results shown in Fig. 2(d) [see also the individual fittings of the MC curves in Fig. 4(a)].

The excellent fidelities of the MC curves in the chaos+impurity model in simulating the experimental ones are discussed more quantitatively as follows. We note that the average value of $r^{2}$ ( \pm standard deviation) among the
25 pairs between the experimental and theoretical $\Delta \sigma(B) \mathrm{s}$ was $0.9858 \pm 0.0083$ for the chaos+impurity model $(|B| \leqslant$ $1.42 \mathrm{mT})$, whereas that for the chaos only model $(|B| \leqslant$ $0.32 \mathrm{mT}$ ) was $0.9885 \pm 0.0090$. More specifically, the difference between the theoretical and experimental $\Delta \sigma\left(\left\langle E_{z}\right\rangle, B\right)$ 's at each $\left(V_{\mathrm{g}}, B\right)$ point was typically $\frac{1}{10}$ of the standard deviation of $\Delta \sigma\left(\left\langle E_{z}\right\rangle, B\right)$ (either theoretical or experimental) using $B$ as the independent variable.

The obtained values of $L_{\phi}$ and $\ell_{\mathrm{ex}}$, as plotted in Figs. 4(c) and 4(d), respectively, are found to be reasonable physically. For example, $L_{\phi}=v_{\mathrm{F}} \tau_{\phi} \approx 250 \mu \mathrm{m}$ and $\ell_{\mathrm{ex}} \approx 0.9 \mu \mathrm{m}$ at $V_{\mathrm{g}}=0.85 \mathrm{~V}$ are consistent with the $\tau_{\phi}$ value estimated at $T_{\mathrm{el}} \approx 100 \mathrm{mK}\left(\sim 3 \times 10^{-10} \mathrm{~s}\right)$ [Fig. $6(\mathrm{~b})$ and $\mathrm{S} 2$ in the Supplemental Material [36]] and the mobility $\mu$ value of the pristine bulk $2 \mathrm{DES}$, respectively. We note that $\ell_{\mathrm{ex}} \approx 0.9 \mu \mathrm{m}$ in the chaos+impurity model is translated to $\mu \approx 4 \mathrm{~m}^{2} / \mathrm{Vs}$ of the bulk mobility, which qualitatively agrees with $\mu \approx 7.6 \mathrm{~m}^{2} / \mathrm{V} \mathrm{s}$ of the pristine bulk 2DES obtained in separate experiments $[16,34,35]$.

The resultant values of $\lambda_{\mathrm{F}}$, as plotted in Fig. 4(b), present a ground for the validity of the present approach. In this regard, we recall the following equation, deduced for the pristine bulk 2DESs [14]:

$$
k_{\alpha}=\frac{\alpha m^{*}}{\hbar^{2}}=\frac{|e| m_{e}}{2 \epsilon_{\mathrm{S}} \epsilon_{0} \hbar^{2}}\left(\frac{a_{\mathrm{SO}} m^{*}}{m_{e}}\right)\left(N_{\mathrm{S}}^{*}-N_{\mathrm{S}}\right),
$$

where the value of the intrinsic constant for the Rashba effect $a_{\mathrm{SO}} m^{*} / m_{e}$ is evaluated to be $1.17 \mathrm{eV}^{2}$ at
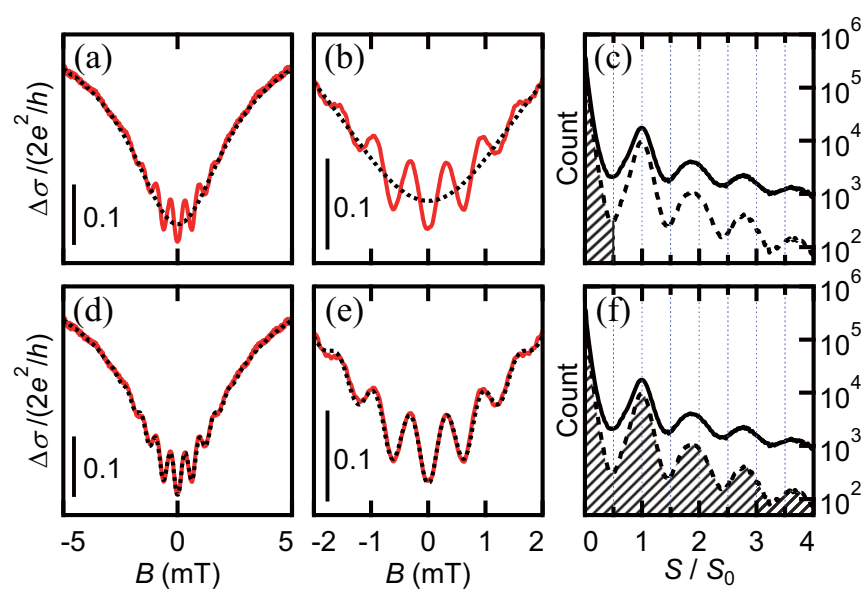

FIG. 5. MC curve of MRA in a wide magnetic field range. (a), (b), (d), (e) Red curves represent the same experimental $\Delta \sigma$ data of MRA taken at $T_{\mathrm{el}} \sim 500 \mathrm{mK}$, where $k_{\alpha}$ is tuned to be zero by $V_{\mathrm{g}}$. (b) and (e) are the magnifications of (a) and (d), respectively, for a narrower magnetic field range. Black dotted curves in (a) and (b) are the $r^{2}$ maximized $\Delta \sigma$ in the "chaos+impurity" model using only a partial set of the closed-loop trajectories that fall in the areal range $S / S_{0} \leqslant 0.5$ [the hatched region in (c)]. Black dotted curves in (d) and (e) are the $r^{2}$ maximized $\Delta \sigma$ in the chaos+impurity model using all the closed-loop trajectories [the hatched region in (f)]. (c), (f) Black solid and dashed curves are the areal distributions of the nominal and weighted return counts, respectively [the latter with $\left.\exp \left(-L_{i} / L_{\phi}\right)\right]$, using $\Delta S=0.05 \mu \mathrm{m}^{2}$ for the histogram bin. Parameter values used in this simulation are $k_{\mathrm{c}}=0.121 \mathrm{rad} / \mu \mathrm{m}$, $\ell_{\mathrm{ex}}=0.94 \mu \mathrm{m}$, and $L_{\phi}=75 \mu \mathrm{m}$. 

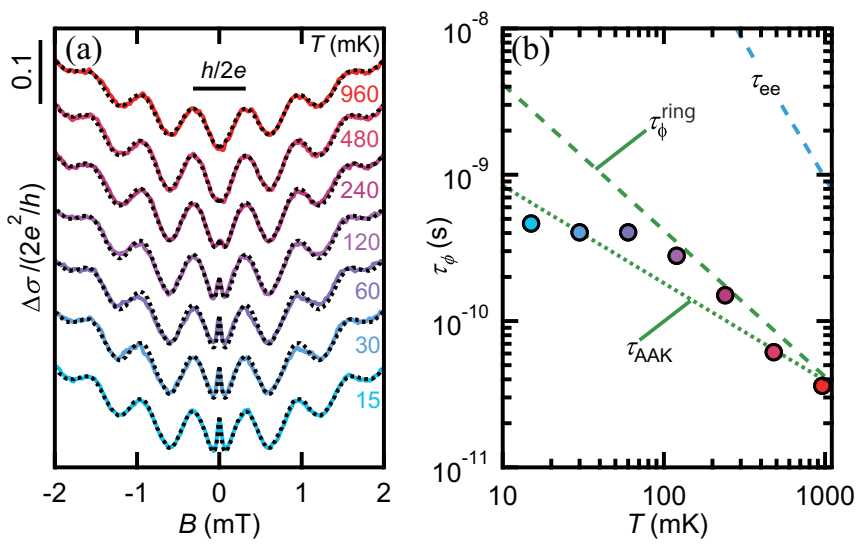

FIG. 6. Temperature dependence of the MC curve in MRA. (a) MC curves at several different $T$ 's. Experimental results are indicated by colored solid curves and the $r^{2}$-maximized simulated results from the "chaos+impurity" model are indicated by black dotted curves. (b) Temperature dependence of the phase coherence times extracted from the chaos+impurity model (colored solid circles). $\tau_{\mathrm{ee}}$, $\tau_{\mathrm{AAK}}$, and $\tau_{\phi}^{\text {ring }}$ [Eqs. (5), (7), and (8)] are plotted by the blue dashed, green dotted, and green dashed lines, respectively.

$N_{\mathrm{S}}=N_{\mathrm{S}}^{*}=1.9 \times 10^{16} \mathrm{~m}^{-2}$, letting $N_{\mathrm{S}}^{*}$ be the carrier density at which $\left\langle E_{z}\right\rangle=0 \mathrm{~V} / \mathrm{m}$ is realized in the pertinent 2DES (sample KH3-1), and $\epsilon_{\mathrm{S}}=13.1$ is the relative dielectric constant for $\mathrm{In}_{0.53} \mathrm{Ga}_{0.47} \mathrm{As}$. Here, $m_{e}$ and $\epsilon_{0}$ are the free-electron mass and dielectric constant in vacuum, respectively. Equation (4) can be used to connect the values between $\lambda_{\mathrm{F}} \equiv \sqrt{2 \pi / N_{\mathrm{S}}}$ and $k_{\alpha}$, which in turn provides the $\lambda_{\mathrm{F}}$ vs $V_{\mathrm{g}}$ relation independently of the present procedure of parameter extraction. The values of $\lambda_{F}$ thus obtained are plotted by the black dotted curve in Fig. 4(b), which are in good agreement with those obtained in the chaos+impurity model.

The limitation of the present model is discussed next. The observed $V_{\mathrm{g}}$ dependencies of $L_{\phi}$ and $\ell_{\mathrm{ex}}$ as in Figs. 4(c) and 4(d), respectively, are only qualitatively physical. Lying at the bottom of the discrepancy is the assumption that the shape and size of the MRA are unchangeable with $V_{\mathrm{g}}$. However, the exact shape of the MRA is of course variable through the gate depletion. Detailed analyses of the classical conductivities $\sigma_{0}$ as a function of $V_{\mathrm{g}}$ may shed light on this issue in the future research. In this work, we report only that our $\sigma_{0}$ (quantitatively equivalent to $\sigma_{0.16 \mathrm{mT}}$ in Sec. IIB) increased monotonically from 6.8 to 14.2 in the unit of $2 e^{2} / h$ for $V_{\mathrm{g}}$ from 0.2 to $1.4 \mathrm{~V}$, respectively, without following the $\sim \sqrt{N_{\mathrm{S}}}$ behavior as expected from the Einstein relation $\sigma_{0}=e^{2} N_{0}\left(E_{\mathrm{F}}\right) D$. Here, the electronic density of states at the Fermi energy $N_{0}\left(E_{\mathrm{F}}\right)$ is constant with $V_{\mathrm{g}}$ and the diffusion constant $D$ is supposed to obey the $\sim \sqrt{N_{\mathrm{S}}}$ behavior with fixed MRA shape and $\ell_{\mathrm{ex}}$.

\section{Origin of the background component in the MC curves}

Plotted in Figs. 5(a) and 5(d) are the MC data of the same MRA sample as above, but with a wider magnetic field range $(|B| \leqslant 5 \mathrm{mT})$, where $k_{\alpha}$ was tuned to be zero by the gate. Although the measurement here was performed at the base temperature of the dilution refrigerator (15 $\mathrm{mK}$ at the mixing chamber), $T_{\mathrm{el}}$ judged from the value
$L_{\phi}=75 \mu \mathrm{m}\left(\tau_{\phi}=8.81 \times 10^{-11} \mathrm{~s}\right)$ turned out to be rather high $(\sim 500 \mathrm{mK})$ because proper low-pass filtering was not employed in the MC measurement. This is why the small WAL peak around $B=0 \mathrm{mT}$ is absent in Figs. 5(a), 5(b), 5(d), and 5(e). Surprisingly, the chaos+impurity model was able to reproduce the experimental results for a magnetic field range as wide as $8 \Delta B$, where $\Delta B=h / 2 e S_{0}$.

Plotted in Figs. 5(c) and 5(f) are the areal histograms of the closed loops generated by the chaos+impurity model, both for nominal count (solid curve) and weighted count with $\exp \left(-L_{i} / L_{\phi}\right)$ (dashed curve). We note that the other quantum modifications, such as $A_{i}\left(k_{\alpha}, k_{\mathrm{c}}\right)$ and $\left(1+\cos \theta_{i}\right)$ as in Eq. (1), are not included in the dashed curve in Figs. 5(c) and 5(f). Discovered clearly here is the fact that the background component of the MC curves with a wide magnetic field range is governed by the closed loops with relatively small encircling areas $\left(S \leqslant 0.5 S_{0}, S_{0}\right.$ being $3.24 \mu \mathrm{m}^{2}$ ) in the chaos+impurity model [black dashed curves in Figs. 5(a) and 5(b)].

\section{Temperature dependence of $\tau_{\phi}$ in MRA}

Let us finally consider the temperature dependence of the MC curves of MRA. For this study, we used the same MRA shape and layer structure as in Figs. 1(b) and 1(d), but the wafer had slightly different doping (the exact wafer used here is sample KH1-3 in Ref. [14]). Shown in Fig. 6(a) are the traces of the MC curves (colored solid curves) obtained at seven different temperatures below $1 \mathrm{~K}$ that are monitored at the mixing chamber, where $k_{\alpha}$ was tuned to be zero by the gate. We note that most of the external conditions in our MRA are temperature independent below $1 \mathrm{~K}$. Only $L_{\phi}$ is highly sensitive to temperature because of the electron phase decoherence in the quasi-2D and one-dimensional (1D) systems $[6,50,51]$. Thus, we analyzed the given MC curves using $L_{\phi}$ as the only variable parameter (black dotted curves) using the chaos+impurity model. The other parameter values used were $k_{\mathrm{c}}=0.150 \mathrm{rad} / \mu \mathrm{m}$ and $\ell_{\mathrm{ex}}=1.3 \mu \mathrm{m}$.

Plotted in Fig. 6(b) are the values of the phase coherent times $\tau_{\phi}$ converted from the extracted $L_{\phi}$ values using $L_{\phi}=v_{\mathrm{F}} \tau_{\phi}$ $\left(N_{\mathrm{S}}=N_{\mathrm{S}}^{*}=1.06 \times 10^{16} \mathrm{~m}^{-2}\right.$ and $\left.m^{*}=0.047 m_{e}[14]\right)$, as a function of temperature measured at the mixing chamber $(T)$. A separate study indicated that the $T_{\mathrm{el}}$ agrees with $T$ only above $100 \mathrm{mK}$ in our system, if proper low-pass filtering is employed (S2 in the Supplemental Material [36]). Consistent with this is the saturation of the $\tau_{\phi}$ values below $T \approx 100 \mathrm{mK}$ in Fig. 6(b). We find the disappearance of the WAL peak around $B=0 \mathrm{mT}$ above $T \sim 480 \mathrm{mK}$ in Fig. 6(a). We can recall our estimation of $T_{\mathrm{el}} \sim 500 \mathrm{mK}$ in Fig. 5, where the WAL peak was not visible either.

Both the $\tau_{\phi}$ values themselves and their dependence on temperature were found to agree with predictions based on the Fermi liquid theory [50,51]. It is known that electronelectron scattering is responsible for $\tau_{\phi}$, not electron-phonon scattering, at low temperatures such as below $1 \mathrm{~K}$ [6]. The electron-electron scattering mechanisms that dominate dephasing consist of large-energy-transfer scattering and smallenergy-transfer (Nyquist) scattering. We denote the decoherence times associated with these mechanisms by $\tau_{\mathrm{ee}}$ and $\tau_{\mathrm{N}}$, respectively, where the total $\tau_{\phi}$ is obtained by $\tau_{\phi}^{-1} \approx \tau_{\mathrm{ee}}^{-1}+\tau_{\mathrm{N}}^{-1}$. 
The expression of $\tau_{\mathrm{ee}}^{-1}$ is given as $[6,50]$

$$
\tau_{\mathrm{ee}}^{-1}=\frac{\pi}{4} \frac{\left(k_{\mathrm{B}} T\right)^{2}}{\hbar E_{\mathrm{F}}} \ln \frac{E_{\mathrm{F}}}{k_{\mathrm{B}} T},
$$

which is plotted with a blue dashed line in Fig. 6(b) using $E_{\mathrm{F}}=54 \mathrm{meV}$ for the Fermi energy. It turned out that $\tau_{\mathrm{ee}}^{-1}$ is negligible relative to $\tau_{\mathrm{N}}^{-1}$ in the investigated temperature range. We have different expressions for $\tau_{\mathrm{N}}^{-1}$ for a diffusive 2DES and a quasi-1D wire system $[50,51]$

$$
\tau_{\mathrm{N}(2 \mathrm{D})}^{-1}=\frac{k_{\mathrm{B}} T}{2 \pi \hbar} \frac{\lambda_{\mathrm{F}}}{\ell} \ln \frac{\pi \ell}{\lambda_{\mathrm{F}}}
$$

and

$$
\tau_{\mathrm{N}(1 \mathrm{D})}{ }^{-1}=\tau_{\mathrm{AAK}}{ }^{-1}=\frac{1}{2}\left(\frac{k_{\mathrm{B}} T}{W} \sqrt{\frac{\pi}{\hbar m^{*}} \frac{\lambda_{\mathrm{F}}}{\ell}}\right)^{2 / 3},
$$

respectively, where $\ell$ and $W$ are the mean-free path in the pertinent system (pristine 2DES or quasi-1D wire) and the width of the quasi-1D wire, respectively. It is noted that a factor $\frac{1}{2}$ in the right-hand side of Eq. (7) is through the time-dependent phase factor which is well established by now [27,52-55]. The apparent agreement between the experimental $\tau_{\phi}$ and theoretical $\tau_{\mathrm{N}(2 \mathrm{D})}$ using unphysical $\ell=21.1 \mathrm{~nm}$ for $T \gtrsim 100 \mathrm{mK}\left[\tau_{\mathrm{N}(2 \mathrm{D})}\right.$ is also given by the green dashed line in Fig. 6(b), the same plot as for $\tau_{\phi}^{\text {ring }}$ of Eq. (8)] was also found in a zero-dimensional (0D) open quantum-dot system $[6,56]$. The more recent (and correct) exposition for the $\tau_{\phi} \sim T^{-1}$ behavior in nontrivial geometries as in the present case is in Refs. [56-62].

Plotted in Fig. 6(b) with the green dotted line is $\tau_{\text {AAK }}$ as a function of temperature, substituting $\ell=85 \mathrm{~nm}$, which is deduced from the experimental $\sigma_{0}$ of the MRA assuming the Einstein relation, and $W=0.5 \mu \mathrm{m}$ in Eq. (7). Here, the values obtained $\left(\tau_{\mathrm{AAK}}\right)$ agree qualitatively well with the experimental results $\left(\tau_{\phi}\right)$ in the given temperature range, although the experimental results did not follow the predicted $T^{-2 / 3}$ dependence in $\tau_{\mathrm{AAK}}$.

Recently, much progress has been made in the study of electron decoherence (by the electron-electron interaction) in complex geometries [57-60] with some verification in experiments $[61,62]$. These studies demonstrate that the decoherence by the electron-electron interaction is geometry dependent. In ring arrays, it has then been shown that, in the high- $T$ regime, i.e., when $\ell_{\phi}$ is smaller than the ring perimeter $L$, electrons following trajectories encircling and not encircling the ring have a $\tau_{\phi} \propto T^{-1}$ and a $\tau_{\phi} \propto T^{-2 / 3}$ dependence, respectively. Within this theoretical framework, the $T^{-1}$ dependence extracted in our study suggests that the dephasing time $\tau_{\phi}$ plotted in Fig. 6 is dominated by contributions from electron going along trajectories encircling the ring and giving rise to the magnetoconductance oscillations. To further analyze our data, we fitted $\tau_{\phi}$ vs $T$ with the $T^{-1}$ law expected for electrons encircling the ring, given by

$$
\tau_{\phi}^{\text {ring }}=\frac{64}{\pi^{3}} \frac{\hbar}{k_{\mathrm{B}}} \frac{\alpha_{d} N_{c} \ell}{L T},
$$

where $N_{c}=\frac{k_{\mathrm{F}} W}{\pi}$ is the number of modes in the rings and $\alpha_{d}$ is a dimension dependent factor. Using $\alpha_{1}=2, W=0.5 \mu \mathrm{m}$ and $L=6.4 \mu \mathrm{m}$, we obtained an elastic scattering length $\ell$ of $167 \mathrm{~nm}$ for $\tau_{\phi}^{\text {ring }}$ in Fig. 6(b). We find that this value $(\ell=$ $167 \mathrm{~nm})$ is in better agreement with $\ell=85 \mathrm{~nm}$ deduced from the experimental $\sigma_{0}$ of the MRA assuming the Einstein relation. We were thus led to similar conclusions as in Ref. [61], despite the apparent differences in the device details aside from their ring structures. Such observation supports the important role of the electron trajectories encircling the rings, as suggested by theories [57-60], in predicting the correct temperature dependence of phase coherent time.

\section{CONCLUSIONS}

We demonstrated an approach of examining electron properties directly from chaotic trajectories in mesoscopic ring array (MRA) structures. In experiments, we measured the gate and temperature dependencies of the magnetoconductivities (MCs) of MRAs that were fabricated in InGaAs quantum wells (QWs), of which the Rashba spin-orbit interaction (SOI) is gate controllable. The gate dependence of the MC curves showed an Onsager symmetry related to the SOI, i.e., $\Delta \sigma\left(\left\langle E_{z}\right\rangle, B\right)=$ $\Delta \sigma\left(-\left\langle E_{z}\right\rangle, B\right)$, which facilitated our semiclassical billiard ball simulation, providing the gate-voltage value that annihilates the Rashba SOI. Applying the real-space formalism of the weak localization/antilocalization effect based on the timereversal interferences on all the chaotic return trajectories in our MRAs, we were able to reproduce the experimental MC curves with high fidelity. Various electron parameters (Fermi wavelength, strength of the residual SOI, probabilistic scattering length, and phase coherence length) in addition to the Rashba SOI were thereby extracted from a single MC curve quantitatively. The approach demonstrated here is applicable to a wide variety of mesoscopic and nanostructured materials and provides a diagnostic tool for investigating the exotic properties of two-dimensional electrons.

\section{ACKNOWLEDGMENTS}

T. Koga, S. Faniel, and A. Sawada are indebted to Professor T. Ando, Professor K. Yakubo, and Professor Y. Niimi for fruitful discussions. The authors are grateful to Dr. A. Subagyo and Professor K. Sueoka for the growth of a $\mathrm{HfO}_{2}$ layer by atomic layer deposition. This work was supported by JSPS KAKENHI Grant No. 16H01045.
[1] R. A. Jalabert, H. U. Baranger, and A. D. Stone, Conductance Fluctuations in the Ballistic Regime: A Probe of Quantum Chaos? Phys. Rev. Lett. 65, 2442 (1990).

[2] C. M. Marcus, A. J. Rimberg, R. M. Westervelt, P. F. Hopkins, and A. C. Gossard, Conductance Fluctuations and Chaotic
Scattering in Ballistic Microstructures, Phys. Rev. Lett. 69, 506 (1992).

[3] H. U. Baranger, R. A. Jalabert, and A. D. Stone, Weak Localization and Integrability in Ballistic Cavities, Phys. Rev. Lett. 70, 3876 (1993). 
[4] A. M. Chang, H. U. Baranger, L. N. Pfeiffer, and K. W. West, Weak Localization in Chaotic versus Nonchaotic Cavities: A Striking Difference in the Line Shape, Phys. Rev. Lett. 73, 2111 (1994).

[5] K. Richter and M. Sieber, Semiclassical Theory of Chaotic Quantum Transport, Phys. Rev. Lett. 89, 206801 (2002).

[6] A. G. Huibers, M. Switkes, C. M. Marcus, K. Campman, and A. C. Gossard, Dephasing in Open Quantum Dots, Phys. Rev. Lett. 81, 200 (1998).

[7] D. M. Zumbühl, J. B. Miller, C. M. Marcus, K. Campman, and A. C. Gossard, Spin-Orbit Coupling, Antilocalization, and Parallel Magnetic Fields in Quantum Dots, Phys. Rev. Lett. 89, 276803 (2002).

[8] O. Zaitsev, D. Frustaglia, and K. Richter, Role of Orbital Dynamics in Spin Relaxation and Weak Antilocalization in Quantum Dots, Phys. Rev. Lett. 94, 026809 (2005).

[9] F. J. Ohkawa and Y. Uemura, Quantized surface states of a narrow-gap semiconductor, J. Phys. Soc. Jpn. 37, 1325 (1974).

[10] Yu. A. Bychkov and E. I. Rashba, Oscillatory effects and the magnetic susceptibility of carriers in inversion layers, J. Phys. C: Solid State Phys. 17, 6039 (1984).

[11] T. Koga, J. Nitta, and M. van Veenhuizen, Ballistic spin interferometer using the rashba effect, Phys. Rev. B 70, 161302 (2004).

[12] T. Koga, Y. Sekine, and J. Nitta, Experimental realization of a ballistic spin interferometer based on the rashba effect using a nanolithographically defined square loop array, Phys. Rev. B 74, 041302 (2006).

[13] T. Bergsten, T. Kobayashi, Y. Sekine, and J. Nitta, Experimental Demonstration of the Time Reversal Aharonov-Casher Effect, Phys. Rev. Lett. 97, 196803 (2006).

[14] S. Faniel, T. Matsuura, S. Mineshige, Y. Sekine, and T. Koga, Determination of spin-orbit coefficients in semiconductor quantum wells, Phys. Rev. B 83, 115309 (2011).

[15] G. Dresselhaus, Spin-orbit coupling effects in zinc blende structures, Phys. Rev. 100, 580 (1955).

[16] S. Mineshige, S. Kawabata, S. Faniel, J. Waugh, Y. Sekine, and T. Koga, Semiclassical interpretation of the spin interference effect observed in square loop arrays of $\operatorname{In}_{0.53} \mathrm{Ga}_{0.47} \mathrm{As} / \mathrm{In}_{0.52} \mathrm{Al}_{0.48} \mathrm{As}$ quantum wells, Phys. Rev. B 84, 233305 (2011).

[17] C. P. Umbach, C. Van Haesendonck, R. B. Laibowitz, S. Washburn, and R. A. Webb, Direct Observation of Ensemble Averaging of the Aharonov-Bohm Effect in Normal-Metal Loops, Phys. Rev. Lett. 56, 386 (1986).

[18] D. Weiss, M. L. Roukes, A. Menschig, P. Grambow, K. von Klitzing, and G. Weimann, Electron Pinball and Commensurate Orbits in a Periodic Array of Scatterers, Phys. Rev. Lett. 66, 2790 (1991).

[19] M. Ferrier, L. Angers, A. C. H. Rowe, S. Guéron, H. Bouchiat, C. Texier, G. Montambaux, and D. Mailly, Direct Measurement of the Phase-Coherence Length in a GaAs/GaAlAs Square Network, Phys. Rev. Lett. 93, 246804 (2004).

[20] R. A. Webb, S. Washburn, C. P. Umbach, and R. B. Laibowitz, Observation of h/e Aharonov-Bohm Oscillations in NormalMetal Rings, Phys. Rev. Lett. 54, 2696 (1985).

[21] F. Schopfer, F. Mallet, D. Mailly, C. Texier, G. Montambaux, C. Bäuerle, and L. Saminadayar, Dimensional Crossover in Quantum Networks: From Macroscopic to Mesoscopic Physics, Phys. Rev. Lett. 98, 026807 (2007).

[22] A. G. Aronov and Yu. V. Sharvin, Magnetic flux effects in disordered conductors, Rev. Mod. Phys. 59, 755 (1987).
[23] G. M. Minkov, A. V. Germanenko, V. A. Larionova, S. A. Negashev, and I. V. Gornyi, Analysis of negative magnetoresistance: Statistics of closed paths. I. Theory, Phys. Rev. B 61, 13164 (2000).

[24] A. Sawada and T. Koga, Universal modeling of weak antilocalization corrections in quasi-two-dimensional electron systems using predetermined return orbitals, Phys. Rev. E 95, 023309 (2017).

[25] M. I. Dyakonov, Magnetoconductance due to weak localization beyond the diffusion approximation: The high-field limit, Solid state Commun. 92, 711 (1994).

[26] A. Cassam-Chenai and B. Shapiro, Two dimensional weak localization beyond the diffusion approximation, J. Phys. I (France) 4, 1527 (1994).

[27] E. Akkermans and G. Montambaux, Mesoscopic Physics of Electrons and Photons (Cambridge University Press, Cambridge, 2007).

[28] E. Ya. Sherman, Random Spin-Orbit Coupling and Spin Relaxation in Symmetric Quantum Wells, Appl. Phys. Lett. 82, 209 (2003).

[29] J. R. Bindel, M. Pezzotta, J. Ulrich, M. Liebmann, E. Ya. Sherman, and M. Morgenstern, Probing variations of the rashba spin-orbit coupling at the nanometre scale, Nat. Phys. 12, 920 (2016).

[30] A. K. Geim and P. Kim, Carbon wonderland, Sci. Am. 298, 90 (2008).

[31] E. McCann, K. Kechedzhi, V. I. Fal'ko, H. Suzuura, T. Ando, and B. L. Altshuler, Weak-Localization Magnetoresistance and Valley Symmetry in Graphene, Phys. Rev. Lett. 97, 146805 (2006).

[32] Q. H. Wang, K. Kalantar-Zadeh, A. Kis, J. N. Coleman, and M. S. Strano, Electronics and optoelectronics of two-dimensional transition metal dichalcogenides, Nat. Nanotechnol. 7, 699 (2012).

[33] A. Ohtomo and H. Y. Hwang, A high-mobility electron gas at the $\mathrm{LaAlO}_{3} / \mathrm{SrTiO}_{3}$ heterointerface, Nature (London) 427, 423 (2004).

[34] T. Koga, T. Matsuura, S. Faniel, S. Souma, S. Mineshige, Y. Sekine, and H. Sugiyama, Beating analysis of shubnikov de haas oscillation in $\mathrm{In}_{0.53} \mathrm{Ga}_{0.47}$ As double quantum well toward spin filter applications, IEICE Trans. Electron. 95, 770 (2012).

[35] S. Faniel, T. Matsuura, S. Mineshige, Y. Sekine, and T. Koga, Weak localization/antilocalization in a nearly symmetric $\mathrm{In}_{0.53} \mathrm{Ga}_{0.47} \mathrm{As} / \mathrm{In}_{0.52} \mathrm{Al}_{0.48}$ As quantum well, AIP Conf. Proc. 1399, 649 (2011).

[36] See Supplemental Material at http://link.aps.org/supplemental/ 10.1103/PhysRevB.97.195303 for the exact MRA shape and the electron temperature $T_{\mathrm{el}}$.

[37] A. G. Huibers, J. A. Folk, S. R. Patel, C. M. Marcus, C. I. Duruöz, and J. S. Harris, Low-Temperature Saturation of the Dephasing Time and Effects of Microwave Radiation on Open Quantum Dots, Phys. Rev. Lett. 83, 5090 (1999).

[38] L. Meier, G. Salis, I. Shorubalko, E. Gini, S. Schön, and K. Ensslin, Measurement of rashba and dresselhaus spin orbit magnetic fields, Nat. Phys. 3, 650 (2007).

[39] M. Kohda and G. Salis, Physics and application of persistent spin helix state in semiconductor heterostructures, Semicond. Sci. Technol. 32, 073002 (2017).

[40] K. Yoshizumi, A. Sasaki, M. Kohda, and J. Nitta, GateControlled Switching between Persistent and Inverse Persistent Spin Helix States, Appl. Phys. Lett. 108, 132402 (2016). 
[41] M. J. van Veenhuizen, T. Koga, and J. Nitta, Spin-orbit induced interference of ballistic electrons in polygon structures, Phys. Rev. B 73, 235315 (2006).

[42] D. Yu. Sharvin and Yu. V. Sharvin, Magnetic-flux quantization in a cylindrical film of a normal metal, Pis'ma Zh. Eksp. Teor. Fiz. 34, 285 (1981) [JETP Lett. 34, 272 (1981)].

[43] B. L. Al'tshuler, A. G. Aronov, B. Z. Spivak, D. Yu. Sharvin, and Yu. V. Sharvin, Observation of the Aharonov-Bohm effect in hollow metal cylinders, Pis'ma Zh. Eksp. Teor. Fiz. 35, 476 (1982) [JETP Lett. 35, 588 (1982)].

[44] L. Onsager, Reciprocal relations in irreversible processes. I., Phys. Rev. 37, 405 (1931).

[45] S. Chakravarty and A. Schmid, Weak localization: The quasiclassical theory of electrons in a random potential, Phys. Rep. 140, 193 (1986).

[46] A. P. Dmitriev, V. Yu. Kachorovskii, and I. V. Gornyi, Nonbackscattering contribution to weak localization, Phys. Rev. B 56, 9910 (1997).

[47] Y. Niimi, Y. Baines, T. Capron, D. Mailly, F.-Y. Lo, A. D. Wieck, T. Meunier, L. Saminadayar, and C. Bäuerle, Effect of Disorder on the Quantum Coherence in Mesoscopic Wires, Phys. Rev. Lett. 102, 226801 (2009).

[48] Y. Niimi, Y. Baines, T. Capron, D. Mailly, F.-Y. Lo, A. D. Wieck, T. Meunier, L. Saminadayar, and C. Bäuerle, Quantum coherence at low temperatures in mesoscopic systems: Effect of disorder, Phys. Rev. B 81, 245306 (2010).

[49] J. J. Krich and B. I. Halperin, Cubic Dresselhaus Spin-Orbit Coupling in 2D Electron Quantum Dots, Phys. Rev. Lett. 98, 226802 (2007).

[50] B. L. Altshuler, A. G. Aronov, and D. E. Khmel'nitskii, Effects of electron-electron collisions with small energy transfers on quantum localisation, J. Phys. C: Solid State Phys. 15, 7367 (1982).

[51] K. K. Choi, D. C. Tsui, and K. Alavi, Dephasing time and one-dimensional localization of two-dimensional electrons in GaAs $/ \mathrm{Al}_{x} \mathrm{Ga}_{1-x} \mathrm{As}$ heterostructures, Phys. Rev. B 36, 7751 (1987).
[52] F. Pierre, A. B. Gougam, A. Anthore, H. Pothier, D. Esteve, and N. O. Birge, Dephasing of electrons in mesoscopic metal wires, Phys. Rev. B 68, 085413 (2003).

[53] G. Montambaux and E. Akkermans, Nonexponential Quasiparticle Decay and Phase Relaxation in Low-Dimensional Conductors, Phys. Rev. Lett. 95, 016403 (2005).

[54] F. Marquardt, J. von Delft, R. A. Smith, and V. Ambegaokar, Decoherence in weak localization. I. Pauli principle in influence functional, Phys. Rev. B 76, 195331 (2007).

[55] J. von Delft, F. Marquardt, R. A. Smith, and V. Ambegaokar, Decoherence in weak localization. II. Bethe-Salpeter calculation of the cooperon, Phys. Rev. B 76, 195332 (2007); J. von Delft, Influence functional for decoherence of interacting electrons in disordered conductors, Int. J. Mod. Phys. B 22, 727 (2008).

[56] M. Treiber, O. M. Yevtushenko, F. Marquardt, J. von Delft, and I. V. Lerner, Dimensional crossover of the dephasing time in disordered mesoscopic rings, Phys. Rev. B 80, 201305 (2009).

[57] M. Treiber, C. Texier, O. M. Yevtushenko, J. von Delft, and I. V. Lerner, Thermal noise and dephasing due to electron interactions in nontrivial geometries, Phys. Rev. B 84, 054204 (2011).

[58] T. Ludwig and A. D. Mirlin, Interaction-induced dephasing of Aharonov-Bohm oscillations, Phys. Rev. B 69, 193306 (2004).

[59] C. Texier and G. Montambaux, Dephasing due to electronelectron interaction in a diffusive ring, Phys. Rev. B 72, 115327 (2005).

[60] C. Texier, P. Delplace, and G. Montambaux, Quantum oscillations and decoherence due to electron-electron interaction in metallic networks and hollow cylinders, Phys. Rev. B 80, 205413 (2009).

[61] M. Ferrier, A. C. H. Rowe, S. Guéron, H. Bouchiat, C. Texier, and G. Montambaux, Geometrical Dependence of Decoherence by Electronic Interactions in a GaAs/GaAlAs Square Network, Phys. Rev. Lett. 100, 146802 (2008).

[62] T. Capron, C. Texier, G. Montambaux, D. Mailly, A. D. Wieck, and L. Saminadayar, Ergodic versus diffusive decoherence in mesoscopic devices, Phys. Rev. B 87, 041307 (2013). 